\title{
SYMMETRIC CAUSTICS AND CURIE'S PRINCIPLE
}

\author{
ALAIN JOETS, AHMED BELAIDI and ROLAND RIBOTTA \\ Laboratoire de Physique des Solides, Université de Paris-Sud, bât. 510 \\ 91405, Orsay cedex, France \\ E-mail: joets@lps.u-psud.fr
}

\begin{abstract}
Physical systems producing caustics may possess symmetries. In that case the relation between the symmetry of the system, considered as a whole, and the symmetry of the caustic follow a very general symmetry principle, the Curie principle. We give various examples of application of the Curie principle to caustics produced by the deflection of light in liquid crystals: the so called squint effect, the visualization of a new type of roll structure, etc. We show also that the Curie principle applies to physical systems having multiple stable states (variants).
\end{abstract}

1. Introduction. Symmetries play an important role in Physics at various levels. The physical laws may be invariant under some groups, e.g. the Galilean group. Boundary conditions or selection of non-linear modes may also impose geometrical symmetries (structures). Moreover the observer may introduce additional symmetries through its experimental procedure. However, whatever their origin or their role, the symmetries are governed by a very general principle, the Curie principle, expressing that the symmetries of the "causes" are contained in the symmetries of the "effects" [5]. The Curie principle has been successfully applied up to now mainly to the crystallography, where it allows to determine which crystallographic groups are compatible with a given physical property of a crystal, for example the pyroelectricity or the piezoelectricity. But its general character allows us to apply it also to other classes of systems, and in particular to systems producing caustics. In that case, the "cause" is the physical system interacting with the light. In the frame of the geometrical optics it is characterized by a light source and by a field of refractive index deviating the rays. The "effect" is the set of the rays deviated by the index field and consequently the caustic formed by the deviated rays.

At the lowest level, the Curie principle is implicitly used to produce experimentally symmetric caustics. For example a drop having the fourfold symmetry has been used to produce the first singularity (of corank 2) possessing a modulus [15]. This experiment has

2000 Mathematics Subject Classification: Primary 78A05; Secondary 58K99.

The paper is in final form and no version of it will be published elsewhere. 
stimulated the local study of the symmetric lagrangian singularities ([7], [8], [9], [17]). On the other hand, caustics produced by a bi-periodic structure in a liquid crystal has been used [10], [14] to check experimentally a global property of caustics in the 3D space, expressed by the Chekanov relation [4]. In this approach the Curie principle is used in the direct way: the symmetry of the cause (drop, liquid crystal) is known and is exploited to impose a symmetry on the effect (the caustic). However in practice, what is known is the observed effect and the characteristics of the cause have to be deduced from the observed effect. In that problem, directed now in the opposite way, the Curie principle plays a key role. It allows us to find (totally or partially) the symmetry of the physical system from the symmetries given by the observation.

In this paper we show several applications of the Curie principle where the produced effect is a caustic (or rather a set of cusp lines). This case corresponds to the important problem of the visualization of some physical field, whatever its nature: a vector field (for example a flow), a tensorial field (for example constraints), etc. It can be called a generalized ombroscopy, the ombroscopy being the flow visualization technique using the weak ray deflection by a thermal gradient.

Before seeing the applications, we have to give some details about the nature of our physical system.

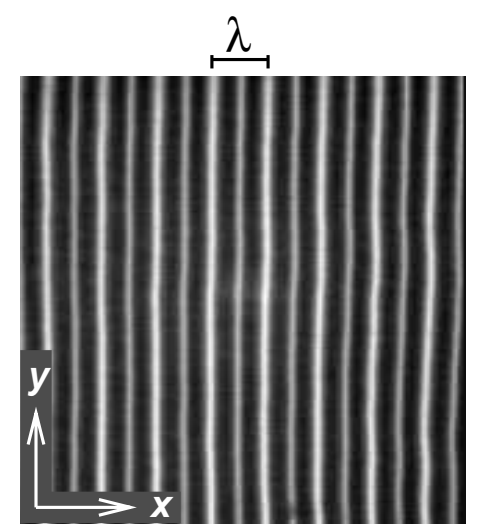

Normal Rolls

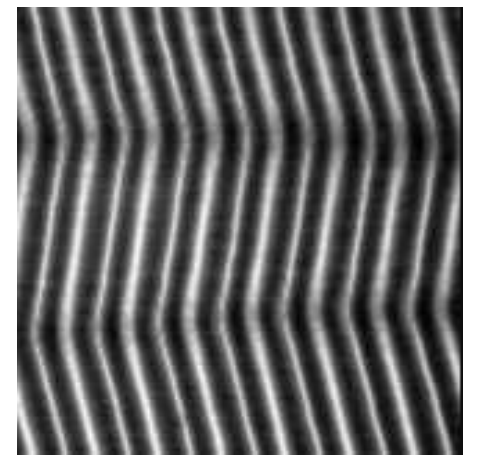

Zig-Zag

Figure 1. Visualization of two roll structures in nematics (normal lighting): the Normal Rolls and the Zig-Zag.

The focal plane cuts the real cusps (straight bright lines).

In both structures the real cusps are equidistant.

The period $\lambda$ of the structure corresponds to 2 rolls.

2. The cause: a roll structure in liquid crystals. In our system, the rays are deflected by a nematic layer. Nematics are liquid crystals, that is to say, organized fluids which are sensitive to various external fields: electric field, magnetic field, temperature gradients, etc. [6]. This property is used to induce convective flows by subjecting a nematic, confined in a layer $-d / 2<z<d / 2$, to a constraint, for instance an alternative electric field. Depending on the values of the constraint, convective structures of different 
(spatial) symmetries are obtained [11]. The most simple structures are the roll structures, consisting of stationary (independent of the time) straight rolls with a diameter nearly equal to the thickness $d \sim 100 \mu \mathrm{m}$ of the layer. From the view point of the symmetries, a roll structure is characterized by two types of translational symmetries: the continuous translations along the roll axis, and the discrete translations normally to the roll axis (period $\lambda$ ) (see Fig. 1). In addition to these translational symmetries, other symmetries may exist.

It is important to realize that the hydrodynamic fields (velocity, pressure gradient) are not directly observable, since the refractive index of a nematic does not depend on these fields. However the nematics are anisotropic materials and the refractive index depends on the local orientation of their rode-like molecules. This orientation is defined by a director, i.e. a unit vector $\vec{n}$, with the convention $-\vec{n} \equiv \vec{n}$. More precisely, the local energy index $N$ for the extraordinary rays is a function of the angle $\beta$ between the ray direction $\vec{r}$ and the director $\vec{n}$ through the relation $N=\sqrt{n_{o} \cos ^{2} \beta+n_{e} \sin ^{2} \beta}$, where $n_{o}$ and $n_{e}$ are respectively the ordinary and extraordinary refractive index [12]. Hereafter the director field is taken as the field defining the structure. For example the standard Normal Rolls structure, the most simple roll structure, is defined by a director lying in the vertical plane $x-z$, normal to the roll axis $y: \vec{n}=(\cos \varphi, 0, \sin \varphi)$, with $\varphi=\varphi_{0} \cos k x \cos \pi z / d$ and $k=2 \pi / \lambda$. We name Normal Rolls structure the structure having this maximal symmetry for a roll structure.

In our experiments the "cause", i.e. the physical system producing the caustic, is thus composed of two elements: the director field $\vec{n}$ (named here roll structure) determining the index field, and the light source. These two elements define completely the caustic produced.

3. The effect: the caustic produced by a roll structure. In the standard experiment, a parallel light beam is sent normally to the nematic layer (normal lighting, along $z$ ), in which a roll structure is set up. Then the rays pass through the layer following trajectories minimizing the optical path $\int N d s$ (Fermat's principle). Since the director is not constant in space, the induced index field continuously deflects the rays and the emerging rays form a congruence of non-parallel rays. This set of lines admits an envelope, the caustic, to which each ray is tangent. Figure 2 shows the caustic produced by the Normal Rolls structure.

The caustic is composed of two parts: a real part (real caustic) located above the sample and a virtual part (virtual caustic) located below, which is the envelope of the prolongation of the emerging rays downwards. In fact the caustic is not disconnected, since its two parts connect at infinity. Note that, due to the continuous invariance of the roll structure, the caustic also possesses a degenerated branch located at infinity. We ignore this branch that is not observable.

The real and virtual parts are composed of a set of cusps, that we name real cusps and virtual cusps (Fig. 2). For the Normal Rolls structure (and normal lighting) the real cusps are located (along $x$ ) above the separations between two adjacent rolls (up-flow and down-flow). The virtual cusps, oriented in the opposite way, are located between the real cusps. 


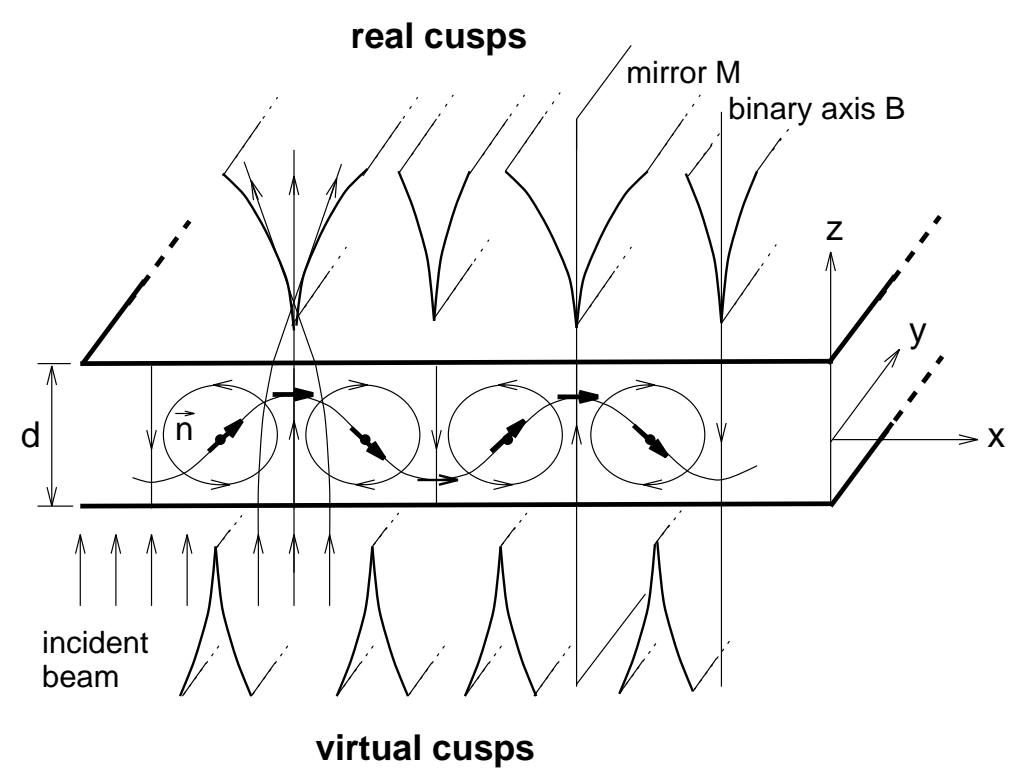

Figure 2. Normal Rolls under normal lighting (along $z$ ). The convective flow is indicated by the thin arrows. The director field $\vec{n}$ (thick arrows) is continuously invariant along the roll axis $y$ and periodic along $x$ (period $\lambda$ ). It is also invariant under the mirrors $M$, the centres of symmetry (black points) and the binary axes $B$.

The visualization of a roll structure simply consists in placing the focal plane (parallel to $x-y$ ) of some optical system (microscope, camera) at some height $z \simeq \pm d$ where it cuts the caustic at or near the real or the virtual cusps. The resulting image is a set of bright straight lines (see Figs. 1, 3, 4 and 5). Roughly speaking, the bright lines formed by the real cusps $(z \simeq d)$ materialize the separation between the rolls and the bright lines formed by the virtual cusps $(z \simeq-d)$ materialize the roll axes.

4. Squint effect, a mechanical or an optical effect? In the Normal Rolls structure the real cusps are equidistant (along $x$ ) since the rolls have the same diameter. On the other hand it is observed that the virtual cusps are not equidistant. They delimit successively thin and thin stripes, the total width of two consecutive stripes being equal to the period $\lambda$ of the structure (see Fig. 3). This apparent asymmetry was named "squint" [1]. Since these cusps appear located below the axes of the rolls, it was concluded that the non-equidistance of the virtual cusps could mean that the Normal Rolls structure is not stable and is replaced by another type of less symmetrical rolls. A mechanical model was constructed to explain the pairing of the rolls by a non-linear mechanism [3].

Let us consider the caustic produced by the Normal Rolls (under normal lighting) from the view point of the symmetries. In addition to the translational symmetries, the Normal Rolls have mirror symmetries, the mirrors being located between two adjacent rolls (see Fig. 2). From the Curie principle, the mirror symmetry must be present in the caustic. Since the real cusps correspond to the separation between rolls, they must be equidistant along the $x$ axis. This characteristic is effectively observed (no squint of the 


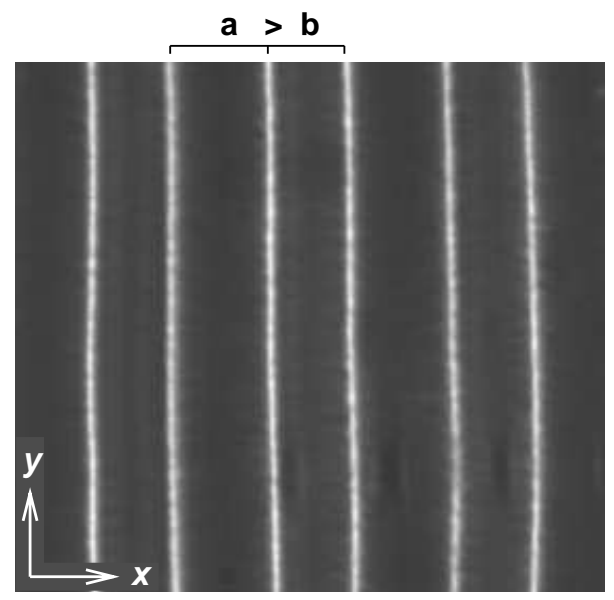

Figure 3. Squint effect. The focal plane cuts the virtual cusps (bright lines).

They are not equidistant along the $x$ axis: $a \neq b$. The period of the pattern is $\lambda=a+b$.

real cusps, see Fig. 1). Of course the mirror symmetry cannot force the virtual cusps to be equidistant, since they do not lie in the mirrors. It remains that the Normal Rolls have also the inversion around their centre leaving the roll axis invariant. However this symmetry cannot be considered as a symmetry of the cause, since it does not belong to the symmetry group of the lighting (parallel rays oriented along $z$ ). Consequently there is no symmetry operation of the cause able to force the equidistance of the virtual cusps. The virtual cusps are expected to generically appear non-equidistant, and they effectively appear so in the experiment. Physically, the squint of the virtual cusps means that the ray passing through a virtual cusp does not pass through a roll axis.

The "squint effect", that is to say, the non-equidistance of the virtual cusps, is totally compatible with the symmetry for the Normal Rolls structure, that is to say, with the maximal symmetry for a roll structure. The squint effect is thus a purely optical effect, observable for any roll structure in nematics.

5. Abnormal Rolls, a new symmetric roll structure. Since a few years, it is suspected that a new type of rolls may exist, the Abnormal Rolls (also called Anomalous Rolls), in which the director is no more confined in the vertical plane $x-z$ but develops now a component along the $y$-axis [2], [16]. Let $\psi$ be the azimuthal angle of the director: $\vec{n}=(\cos \phi \cos \psi, \cos \phi \sin \psi, \sin \phi)$. The Normal Rolls structure is characterized by $\psi=0$ and the Abnormal Rolls, in the most simple model, by $\psi=\psi_{0} \cos \pi z / d$. The important question is to experimentally recognize this new type of rolls. Here too, Curie's principle is very useful.

With respect to the Normal Rolls, the Abnormal Rolls are less symmetrical. In particular they do not have the symmetry through the vertical mirrors $M$. May we conclude, using the same reasoning as in the preceding section, that the real cusps must now appear non-equidistant? No, because here again the cause has symmetry elements, the vertical binary axes $B$ passing between adjacent rolls (see Fig. 1), which force the equidistance of 
the real cusps. In other words, under normal lighting, the Normal Rolls and the Abnormal Rolls do not appear qualitatively different.

The Curie principle now suggests a new type of lighting, breaking the symmetries around the binary axes $B$. Consider for instance an incident beam direction $\vec{r}$ making some angle $\gamma$ with respect to the $z$-axis: $\vec{r}=(0, \sin \gamma, \cos \gamma)$. When $\gamma=0$, we recover the normal lighting for which the Abnormal Rolls do not qualitatively differ from the Normal Rolls. When $\gamma \neq 0$ (oblique lighting), two cases have to be considered. First, when $\psi=0$ (Normal Rolls), the lighting and the director field are invariant with respect to the mirrors $M$, and the real cusps must be equidistant. Then, when $\psi \neq 0$ (Abnormal Rolls), there is no more symmetry element $M$ or $B$ passing through the real cusps and they must appear non-equidistant: squint on the real cusps. The oblique lighting is thus well adapted for the experimental recognition of the Abnormal Rolls. Figure 4 presents rolls under oblique lighting. The real cusps appear clearly non-equidistant. We may conclude that they are not symmetrical around the axes $B$ and that they have necessarily a component $n_{y}$. They are Abnormal Rolls. The normal lighting, usually used in the experiments, prevented during a long time the recognition of this new roll structure.
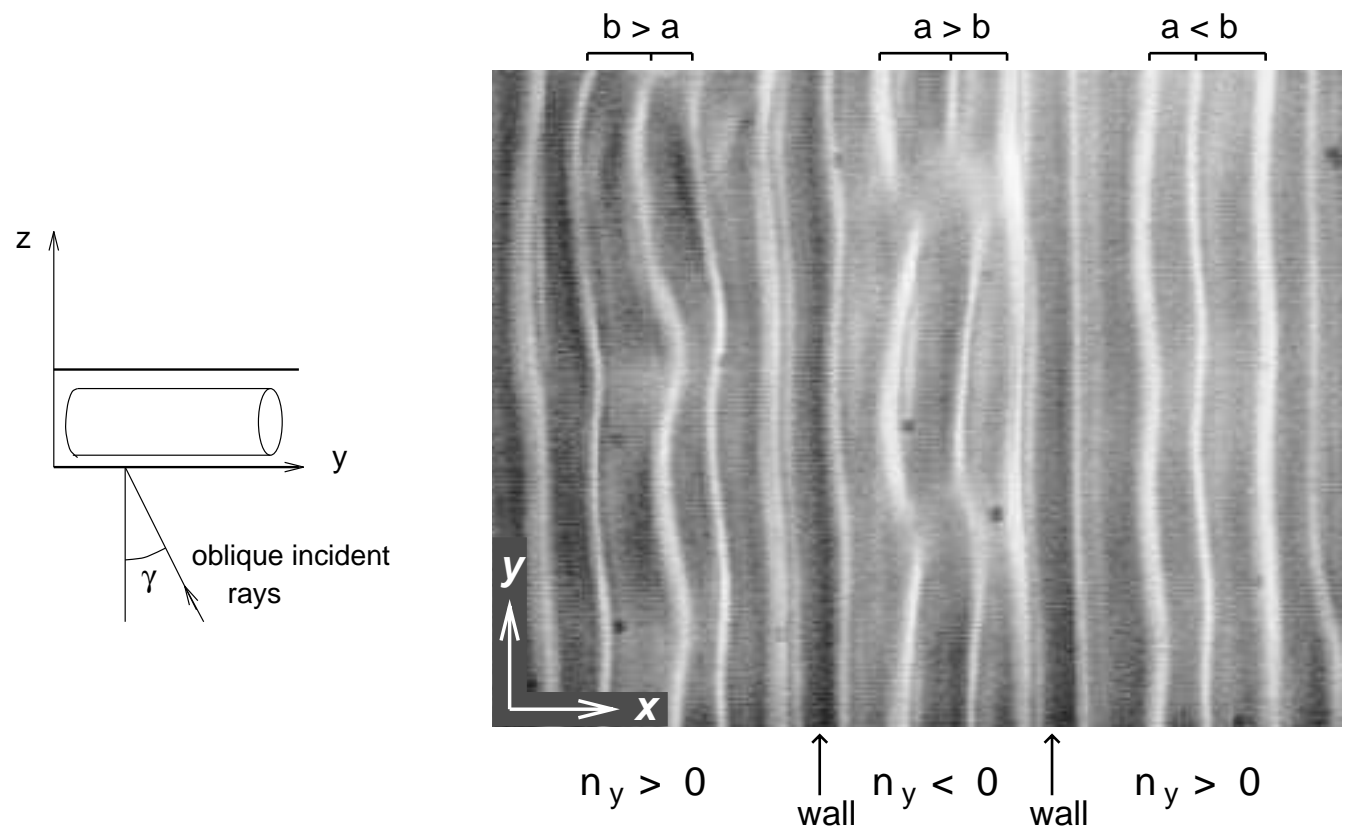

Figure 4. Abnormal Rolls. The incident beam makes an angle $\gamma$ with respect to the vertical $z$ axis (oblique lighting). The focal plane is cuts the real cusps (bright lines) which appear non-equidistant.

The amplitude $\psi_{0}$ of the $n_{y}$ component in the Abnormal Rolls structure, may be positive or negative. Both possibilities are equivalent. As a consequence, the rolls form domains separated by topological domains (walls) (see Fig. 4, where one sees three domains). 
6. Symmetries of the Zig-Zag, a multiple states system. The Curie principle applies also to systems admitting more than one stable state (variants) resulting from a symmetry breaking bifurcation. In that case each particular state has less symmetries than the system before the bifurcation; but the "lost" symmetries are still present: they exchange the different states.

Let us consider the Zig-Zag structure (see Fig. 1). It is composed of two variants, the Zig and the Zag, each variant being a roll structure making an angle $\pm \theta$ with respect to the $y$ axis. The broken symmetries at the Normal Rolls $\rightarrow$ Zig-Zag bifurcation are the mirrors normal to the $x$ and $y$ axes. The vertical binary axes $B$ are not broken and they force the real cusps to appear equidistant under normal lighting (Fig. 1). Of course, the mirrors normal to $x$ and $y$ exchange the Zig and the Zag states.

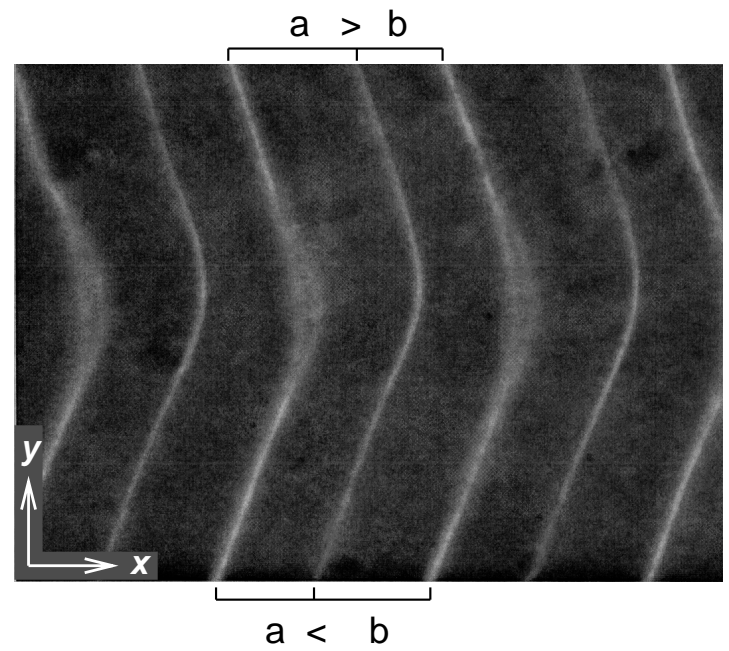

Figure 5. Zig-Zag structure under oblique lighting. The focal plane cuts the real cusps (bright lines). Their relative spacing ( $a$ and $b$ ) changes when one passes from the Zig to the Zag variant.

When the oblique lighting is used, the incident rays are no more invariant around the axes $B$ and according to the Curie principle the real cusps must appear non-equidistant in each variant. We see in Fig. 5 that they effectively delimit successively thin and thick stripes. However a striking feature must be noted if we compare the two variants: a thin stripe in the Zig becomes thick in the Zag and vice versa (Fig. 5). Here again we have to invoke symmetry reasons. Among the symmetries exchanging the two states, the oblique lighting breaks only the mirror normal to $y$, but it conserves the mirror normal to $x$. Let us see the effect of that mirror. Suppose that the small stripe in the Zig variant corresponds to the separation between the up-flow and the down-flow in that order along $x$. By the mirror symmetry this stripe, in the Zag variant, corresponds to a up-flow and down-flow in that order in the $-x$ direction. In other words, a thin stripe becomes a thick stripe when one passes from the Zig to the Zag. This mirror symmetry, lost in the bifurcation producing the Zig-Zag structure, explains the opposite squints on the real cusps in the two variants. 
7. Conclusion. We studied global configurations of symmetrical caustics produced by the deflection of the light through a nematic layer. Their symmetries may be understood by using a general symmetry principle, the Curie principle, relating the symmetries of the structure (the nematic director field) to the symmetries of the caustic. Ignoring this principle may lead to rough misinterpretations of experimental results, for instance the squint effect interpreted as a symmetry breaking. On the other hand, the same principle may be used to recognize new types of structures, for example the Abnormal Rolls structure in which the director has now a component along the roll axis. Finally we have shown on the case of the Zig-Zag structure how it also applies to multiple states systems.

\section{References}

[1] J. S. Barley, E. Gelerinter, S. I. Ben-Abraham, Observation of squint-A nonlinear feature of flow patterns in nematics, Mol. Cryst. Liq. Cryst. 87 (1982), 251-257.

[2] A. Belaidi, Ph.D. thesis, Univ. Paris XI, Orsay, France, 1997.

[3] S. I. Ben-Abraham, Squint-A nonlinear feature of flow patterns in nematics, J. Physique 40 (1979), C3-259-262.

[4] Yu. V. Chekanov, The caustics of geometric optics, Funktsional. Anal. i Prilozhen. 20:3 (1986), 66-69 (Russian); English transl.: Funct. Anal. Appl. 20 (1986), 223-226.

[5] P. Curie, Sur les symétries dans les phénomènes physiques, symétrie d'un champ électrique et d'un champ magnétique, J. de Phys. Théor. et Appl., 3e série (1894), 393-415.

[6] P.-G. de Gennes, J. Prost, The Physics of Liquid Crystals, Clarendon Press, Oxford, 1993.

[7] S. Janeczko, Singularities of Lagrangian varieties and related topics, Acta Appl. Math. 25 (1991), 21-57.

[8] S. Janeczko, M. Roberts, Classification of symmetric caustics. I. Symplectic equivalence, in: Singularity Theory and its Applications, Part II, M. Roberts and I. Stewart (eds.), Lecture Notes in Math. 1463, Springer, Berlin, 1991, 193-219.

[9] S. Janeczko, M. Roberts, Classification of symmetric caustics. II. Caustic equivalence, J. London Math. Soc. (2) 48 (1993), 178-192.

[10] A. Joets, Topology and geometry of caustics in relation with experiments, in: Geometry and Topology of Caustics - Caustics '98, S. Janeczko and V. Zakalyukin (eds.), Banach Center Publ. 50, Warsaw, 1999, 169-177.

[11] A. Joets, R. Ribotta, Hydrodynamic transitions to chaos in the convection of an anisotropic fluid, J. Physique 47 (1986), 595-606.

[12] A. Joets, R. Ribotta, A geometrical model for the propagation of rays in an anisotropic inhomogeneous medium, Optics Communications 107 (1994), 200-204.

[13] A. Joets, R. Ribotta, Caustics and symmetries in optical imaging. The example of convective flow visualisation, J. Phys. I France 4 (1994), 1013-1026.

[14] A. Joets, R. Ribotta, Experimental determination of a topological invariant in a pattern of optical singularities, Phys. Rev. Lett. 77 (1996), 1755-1758.

[15] J. F. Nye, The catastrophe optics of liquid drop lenses, Proc. Roy. Soc. London Ser. A 403 (1986), 1-26.

[16] E. Plaut, W. Decker, A. G. Rossberg, L. Kramer, W. Pesh, A. Belaidi, R. Ribotta, New symmetry breaking in nonlinear electroconvection of nematic liquid crystals, Phys. Rev. Lett. 79 (1997), 2367-2370.

[17] M. Roberts, V. M. Zakalyukin, Symmetric wavefronts, caustics and Coxeter groups, in: Singularity Theory, D. T. Lê, K. Saito and B. Teissier (eds.), World Sci. Publ., River Edge, 1995, 594-626. 